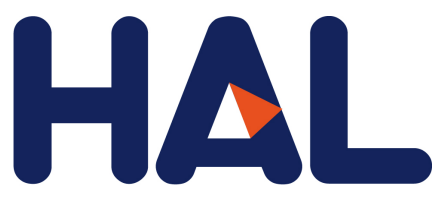

archives-ouvertes

\title{
Thorough Investigation of multipath Techniques in RPL based Wireless Networks
}

\author{
Ana Estrin, Tomas Lagos Jenschke, Georgios Papadopoulos, J Ignacio \\ Alvarez-Hamelin, Nicolas Montavont
}

\section{- To cite this version:}

Ana Estrin, Tomas Lagos Jenschke, Georgios Papadopoulos, J Ignacio Alvarez-Hamelin, Nicolas Montavont. Thorough Investigation of multipath Techniques in RPL based Wireless Networks. IEEE ISCC 2020, Jul 2020, Rennes, France. hal-02870764

\section{HAL Id: hal-02870764 \\ https://hal.archives-ouvertes.fr/hal-02870764}

Submitted on 16 Jun 2020

HAL is a multi-disciplinary open access archive for the deposit and dissemination of scientific research documents, whether they are published or not. The documents may come from teaching and research institutions in France or abroad, or from public or private research centers.
L'archive ouverte pluridisciplinaire HAL, est destinée au dépôt et à la diffusion de documents scientifiques de niveau recherche, publiés ou non, émanant des établissements d'enseignement et de recherche français ou étrangers, des laboratoires publics ou privés. 


\title{
Thorough Investigation of multipath Techniques in RPL based Wireless Networks
}

\author{
Ana Czarnitzki Estrin ${ }^{\dagger}$, Tomas Lagos Jenschke ${ }^{\dagger}$, Georgios Z. Papadopoulos ${ }^{\dagger}$, \\ J.Ignacio Alvarez-Hamelin ${ }^{\ddagger, *}$ and Nicolas Montavont ${ }^{\dagger}$ \\ $\dagger$ IMT Atlantique, Irisa, France, Email: \{firstname.lastname\}@imt-atlantique.fr \\ * Universidad de Buenos Aires, Argentina, Email: ihameli@cnet.fi.uba.ar \\ * CONICET, Argentina
}

\begin{abstract}
With the growth of the Internet of Things appliances in industrial environments, known as Industry 4.0, wireless multihop network solutions have been attracting more attention in the past years. The IPv6 Routing Protocol for Low-Power and Lossy Networks (RPL) is the de facto protocol for Low-power and Lossy Networks specially designed for industrial use-cases. However, the default operation of RPL does not provide a high level of network reliability and low jitter performance. The use of IEEE Std 802.15.4-2015 Time Slot Channel Hopping (TSCH) at the Medium Access Control layer can mitigate the effects of external interference by re-transmitting over different radio frequency. Still, this standard does not support possible link failures or node over-the-air programming. In this paper, we propose the use of multiple disjoint paths to enforce reliability and availability when both RPL and TSCH standards are used. Indeed, we propose and compare two approaches based on the principle of replication: i) packet replication at the source node only, and ii) packet replication at the source node and scattering if two replicas merge. We implemented these two algorithms in Contiki OS and evaluated their trade-offs over the simulated network environment provided by COOJA. Finally, we compare these solutions against the stateof-the-art Packet Automatic Repeat reQuest (ARQ), Replication and Elimination (RE), and Overhearing (PAREO) technique that proposes a braided multipath pattern.
\end{abstract}

Index Terms-Internet of Things, IoT, Industrial IoT, LLNs, RPL, IEEE 802.15.4, TSCH, RAW, PAREO, multipath Routing

\section{INTRODUCTION}

Industry 4.0 refers to highly automated production chains that offer high adaptability and control. For instance, smart factories rely on small sensor devices that measure the leading indicators of the different stages of the production chain. They send those values to remote servers to process them using machine learning methods, proposing decisions based on those results. Due to the necessity of portability and fast adaptability at a low cost, wireless and multi-hop networks are presented as a desirable choice.

This model is applicable in many industries, thanks to the use of Internet of Things (IoT) technologies. These technologies cover different physical objects that can connect their internal networks with the Internet without human interaction. IoT devices can be very different from one to another but are often characterized by limited power, memory, and processing resources. These devices construct Low-power and Lossy
Networks (LLNs), which can operate in various environments [1]. One of the well-known standards for such wireless networks is IPv6 Routing Protocol for Low-Power and Lossy Networks (RPL) [2]. In RPL, one device acts as an LLN border router, connecting the IoT devices with IPv6 to the Internet. RPL is a multi-hop routing protocol, using an Objective Function (OF) [3] to select a parent from a Parent Set (PS), forming a Destination-Oriented Directed Acyclic Graph (DODAG).

At the Medium Access Control (MAC) layer, the IEEE Std 802.15.4-2015 Time Slot Channel Hopping (TSCH) [4] protocol allows reducing the number of collisions, bounding the medium access delay, as well as mitigating external interference by using a channel hopping scheme to transmit over different radio frequencies.

Still the difficulty resides in applying wireless technology to the production chain environment since the industry requires qualityof-service solutions, while the presented protocols are basically best-effort approaches. The default operations of the presented protocols do not take into account temporary link failures or node updates. To tackle this issue, multipath routing with intelligent scheduling has been proposed in the past [5], [6], [7].

In this document, we explore several multipath strategies to understand the trade-offs between additional cost in terms of traffic overhead and network performance. A source node can select $n+$ 1 parents instead of just one (the default RPL operation), allowing the use of $n+1$ disjoint paths in the wireless network. Then, under such a scenario, we investigate the following two techniques:

1) Packet replication at the source node only, where this node sends $\mathrm{n}$ replicas plus the original copy. As a result, we get a total of $n+1$ copies, one to each of its $n+1$ selected parents. The rest of the nodes forwards the received packet to their Preferred Parent (PP).

2) Packet replication at the source following the previous proposal and scattering at the nodes where paths are merged, when two or more relay nodes select a common PP.

We implemented these algorithms over Contiki OS $^{1}$ and performed a series of simulations using the COOJA simulator. Finally, we compared these solutions against the state-of-the-art Common Ancestors (CAs) algorithms that propose braided multipath patterns [8].

\footnotetext{
${ }^{1}$ https://github.com/contiki-os/contiki
} 


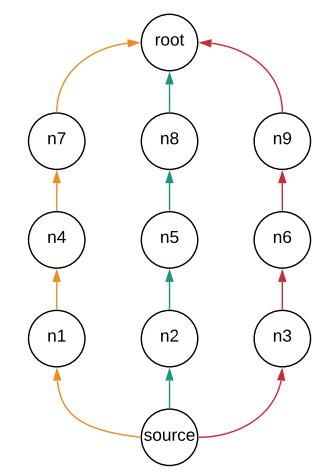

(a) An example of a default scenario.

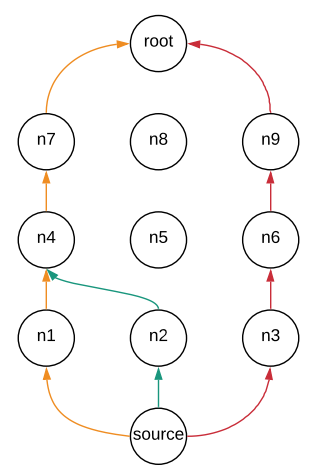

(b) An example of a merging paths scenario.

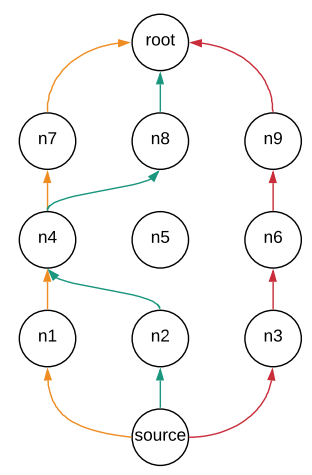

(c) An example of a controlled scenario.

Fig. 1: An example of 3-disjoint paths in a multi-hop topology.

\section{TECHNICAL BACKGROUND}

\section{A. IEEE Std 802.15.4-2015 TSCH}

TSCH is a MAC layer protocol that combines Time-Division Multiple Access (TDMA) and Frequency-Division Multiple Access (FDMA) to provide reliable communication. This protocol divides time into intervals called timeslots, which are grouped into a slot-frame that is repeated during the lifetime of the network. The transmission and reception instances are allocated in cells that correspond to a channel offset within a specific timeslot. TSCH uses Enhanced Beacons (EBs) control packets that are periodically transmitted to keep the schedule synchronized. The cells are classified into two categories:

- Shared cells: the contention-based cells that are assigned for control traffic.

- Dedicated cells: the contention-free cells that are dedicated to data traffic.

Finally, the TSCH schedule can be either centralized, where a single device is selected as a coordinator for the entire network, or distributed, where each node makes its decisions locally in collaboration with its neighbors. In this paper, a centralized scheduler is used.

\section{B. $R P L$}

RPL is a distance vector routing protocol specially designed for LLNs.

This protocol allows each node to select a default parent, building a Destination-Oriented Directed Acyclic Graph ( DODAG), toward a sink called the DODAG Root. The parent selection is determined by an OF [9], which receives one or more metric parameters, providing a rank that represents the distance between the node and its DODAG Root. An example of a metric is the Expected Transmission Count (ETX), which is the default metric. The ETX allows choosing the route with the best link statistics. RPL uses the following three control packets to build the DODAG.

- DODAG Information Object (DIO): it is transmitted in broadcast, and it is used to update and maintain the status of the DODAG.

- Destination Advertisement Object (DAO): it is transmitted each time a node selects a new parent to propagate reverse routing information.
- DODAG Informational Solicitation (DIS): it is transmitted in broadcast to receive a DIO packet in response.

Each node may consider its neighbors with a lower rank than its as a potential parent, grouping them into a Parent Set (PS). In default RPL, one of the nodes in the PS is selected as the default parent, or PP (e.g., the one that provides the lower rank). In our work, we use this PS to select more than one parent and initiate multipath by sending it to multiple parents.

\section{Replicas and Retransmissions}

Since RPL is a multi-hop routing protocol, its Packet Error Rate (PER) is given by a binomial distribution per hop. This protocol uses retransmissions to increase its reliability, which in turn, increases the delay and jitter, since more slot-frames may be required. Because of that, we propose to send multiple copies of a packet (called replicas) to anticipate losses. This work aims to compare the compensation between the use of packet replicas and retransmissions. Through replication, the goal is to transmit copies of the same data packet through different paths. Retransmissions, on the other hand, consist of reinforcing the transmission of a data packet to the same destination when a previous transmission failed, i.e., an acknowledgment was not received. Therefore, the number of replicas determines the number of copies per data packet transmitted over $n$ destinations, while retransmissions define the maximum number of transmission opportunities per data packet from a source node to a destination node.

\section{MULTIPATH STRATEGIES}

In this work, we implemented two different algorithms for achieving n-Disjoint paths. The first one is called " $n$-Disjoint paths: default scenario", where only the source node transmits $n$ copies plus the original data packet to $n+1$ parents. The second algorithm is called " $n$-Disjoint paths: controlled scenario", after that a potential path merging, the "merging point" node forwards each received replica to a different parent. Moreover, we present the Packet Automatic Repeat reQuest (ARQ), Replication and Elimination (RE), and Overhearing (PAREO) functions in this section, since we compare the n-Disjoint strategies against them. 


\section{A. n-Disjoint Paths: Default Scenario}

In the default scenario, only the source node transmits multiple replicas of the same data packet in disjoint paths. The source node selects the $n+1$ best parents given from its PS, where $n$ is the number of replicas and sends a copy of the packet to the parents. The rest of the relay nodes forwards the received packets to their PPs till the DODAG root. Note that this is valid for $n<|P S|$. The ideal case of this implementation, where all replicas follow completely disjoint paths and reach the final destination, is illustrated in Fig. 1a.

However, two or more disjoint paths may merge on one relay node, as that node may be selected as the PP by several nodes. As a result, it is probable that fewer paths (less than $n+=1$ ) are actually used. Fig. 1b is illustrating this case: even without transmission loss, the root may not receive $n+1$ replicas, since some paths may merge. Nodes $n 1$ and $n 2$ have the same PP $n 4$, and thus only one replica is sent from $n 4$. The next algorithm addresses this issue to maintain the number of paths in the network.

\section{B. n-Disjoint Paths: Controlled Scenario}

To overcome such issues, we implemented a second algorithm that detects when this merging takes place and allows the copies to follow different paths. If a relay node receives two or more copies of the same packet from different children, it forwards them to different parents. This technique handles path merging scenarios; however, it does not recreate replicas of packets that were lost due to network quality issues. Fig. 1c shows this scenario, where the node $n 4$ forwards the two received copies to nodes $n 7$ and $n 8$.

In this implementation, each of the relay nodes selects $n+1$ parents from its PS, and stores them in ETX order, where the first would be the PP. If a replica is received at the relay node, after the original copy, it forwards it to the next best parent that was previously stored. As in the Default Scenario, this is valid for $n<|P S|$. d

\section{PAREO}

This technique proposes that each of the nodes selects a second parent, called the Alternative Parent (AP), in addition to the PP. When a data packet is received, the relay node forwards it to both parents [7].

PAREO uses the following functions to ensure maximum reliability and availability, and low jitter performance:

- Automatic Repeat reQuest (ARQ): this function performs a retransmission of data packets if the previous transmission fails.

- Replication and Elimination (RE): this refers to the replication function to send the data packet both to the PP and to the AP. Furthermore, since the replication mechanism introduces additional traffic, PAREO adds an elimination function to discard the unnecessary copies.

- Overhearing $(\mathrm{OH})$ : this technique takes advantage of the shared property of the wireless medium. When the PP and the AP are close enough, then the first can overhear the transmission for the second one and vice-versa.

There are several different algorithms for choosing an AP through common ancestors. The trade-offs between them involve the probability of having an AP versus the number of packets that
TABLE I: Simulation parameters.

\begin{tabular}{lll}
\hline Parameter & n-Disjoint & PAREO (Medium CA) \\
\hline MAC layer retransmissions & $0,1,3,7$ & 1 \\
Replicas & $0,1,2,3,4,5$ & - \\
Link quality & $50 \%, 75 \%$ & $50 \%, 75 \%$ \\
Number of seeds (execution) & 20 & 20 \\
Number of packets per seed & 250 & 250 \\
\hline
\end{tabular}

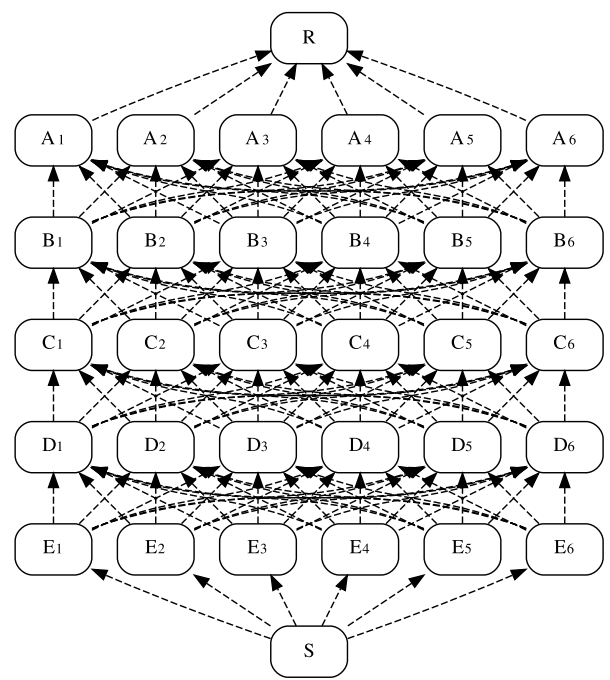

Fig. 2: Network topology used in the experimental evaluations.

traverse the network. In this paper, for the simulations performed, we used the Medium CA method that states that a Parent Candidate (PC) can be selected as an AP of a node $\lambda$ if the PP of the $\mathrm{PP}$ of $\lambda$ is in the PS of the PC [8], i.e., if $P P(\lambda) \in P S(P C)$.

\section{Simulation Setup}

We performed a thorough evaluation of the different configurations to analyze the trade-offs between using only replications, only retransmissions, or a combination of both for the two proposed n-Disjoint algorithms against the PAREO technique. The detailed configuration of the evaluated algorithms is presented in Table I. For each routing algorithm choice, we set different link qualities, $50 \%$ and $75 \%$, between all the nodes. We employed the Directed Graph Radio Medium (DGRM) model that is implemented in COOJA. We chose these values following the Dust Networks ${ }^{2}$ definition of a healthy network where each device should have at least a $50 \%$ link quality with its neighbors. Finally, all three techniques are implemented on top of the Contiki OS, where the simulations were done with COOJA.

\section{A. Topology}

The network topology is illustrated in Fig. 2. It has a total of 32 nodes, where $\mathrm{S}$ is the source node and $\mathrm{R}$ the destination node, i.e., the DODAG root. Each one of the intermediate nodes has 5 neighbors (e.g., $E 1_{1} \ldots E_{6}$ ), forming a total of 5 levels $(A \ldots E)$. The range of radio communication of a node is determined by the topology itself: it communicates with those below and above his level. For example, nodes from the level $D$ are within range of those in levels $E$ and $C$, respectively.

\footnotetext{
${ }^{2}$ SmartMesh IP Application Notes, Linear Technology Corp. 2012-2016.
} 

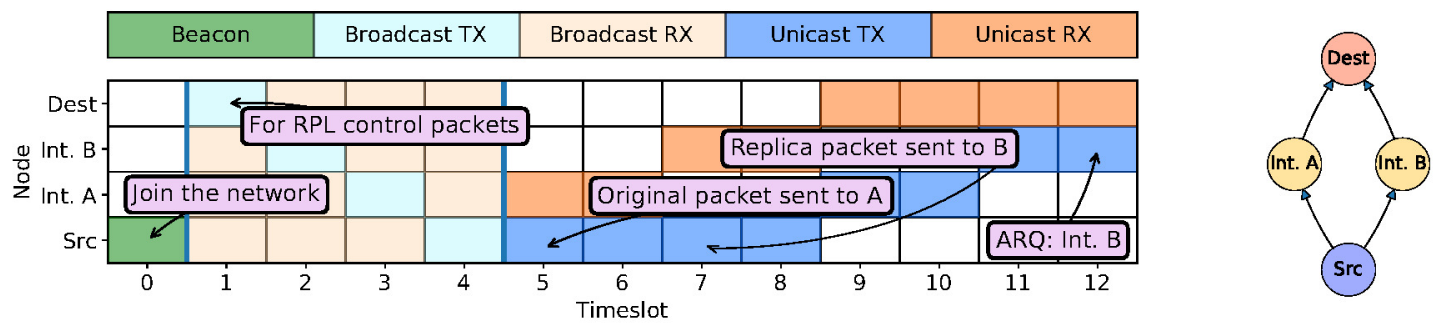

Fig. 3: TSCH example schedule showing replications (one) and ARQ operations.
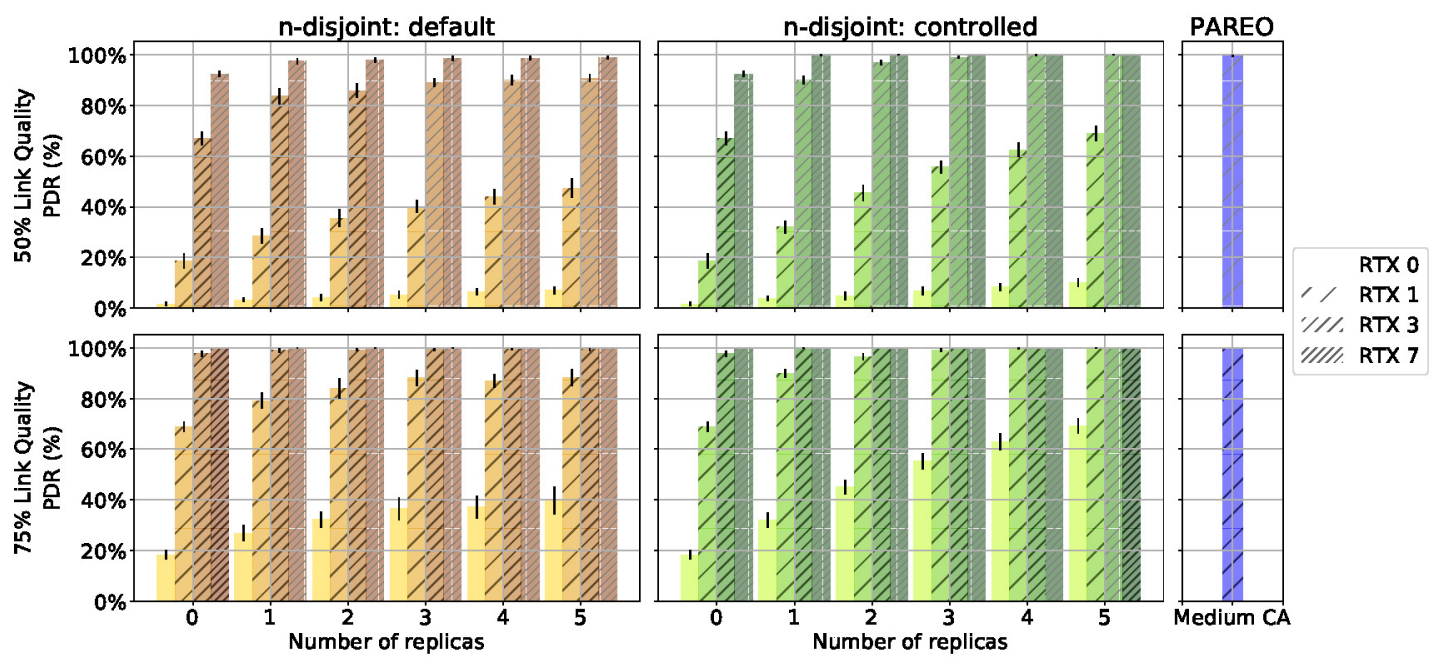

Fig. 4: Packet Delivery Ratio.

This topology was chosen to have multiple parallel paths to evaluate the impact of multipath algorithms. For instance, sending five replicas may result in up to 6 disjoint paths, i.e., five replicas and the original data packet.

\section{B. Schedule}

We used a static and centralized schedule that operates over a single channel. Since TSCH is agnostic of RPL and because our schedule is static, the schedule has assigned timeslots even for links that the routing protocol does not use.

Each node has two timeslots for each of its neighbors. We used this setup to keep the slot-frame as small as possible while having an extra timeslot for retransmissions. In this configuration, the second one is sent on the next timeslot if the first transmission fails. If that fails as well (and if the number of retransmissions allows it), the third one is scheduled in the next slot-frame. This behavior is repeated each time retransmission is required. An example schedule for a smaller topology can be seen in Fig. 3. The number of retransmissions was selected to show the impact that our schedule has on delay and jitter metrics.

\section{Performance evaluation}

\section{A. Simulation Results}

1) Packet Delivery Rate (PDR): We define as PDR the number of packets successfully received from the total amount of packets. We only take into consideration the first copy received from a packet.

The results in Fig. 4 shows that if we increase the number of replicas, the PDR is increased linearly for the n-Disjoint algorithms. This increase can also be seen when using retransmissions as well. The same behavior happens both for $75 \%$ or $50 \%$ link qualities. These values are consistent with the concept that with each replica we transmit at the source node, we are adding exactly one more opportunity for a single data packet to reach its final destination. We can also observe that the controlled scenario has a better PDR than the default one, showing that our topology has some nodes with the same PP, resulting in merging some paths.

Finally, to obtain the same performance of PAREO in the case of $75 \%$ link quality, the n-Disjoint solution needs at least 4 replicas to obtain the same PDR when one retransmission is allowed, or use the controlled n-Disjoint with 0 replica and 7 retransmissions. However, if the link quality is $50 \%$, there is no number of studied replicas that can achieve a 100\% PDR with single retransmission, like PAREO and controlled n-Disjoint for 1 replica and 7 retransmissions. PAREO demonstrates the advantage of using the $\mathrm{OH}$ function for reliability, adding more chances for a data packet to arrive at the DODAG Root.

2) Delay and Jitter: The delay is calculated as the difference between the time of the first transmission and the first replica arrival time, only for originally received packets.

Our performance evaluation results show that the delay and 


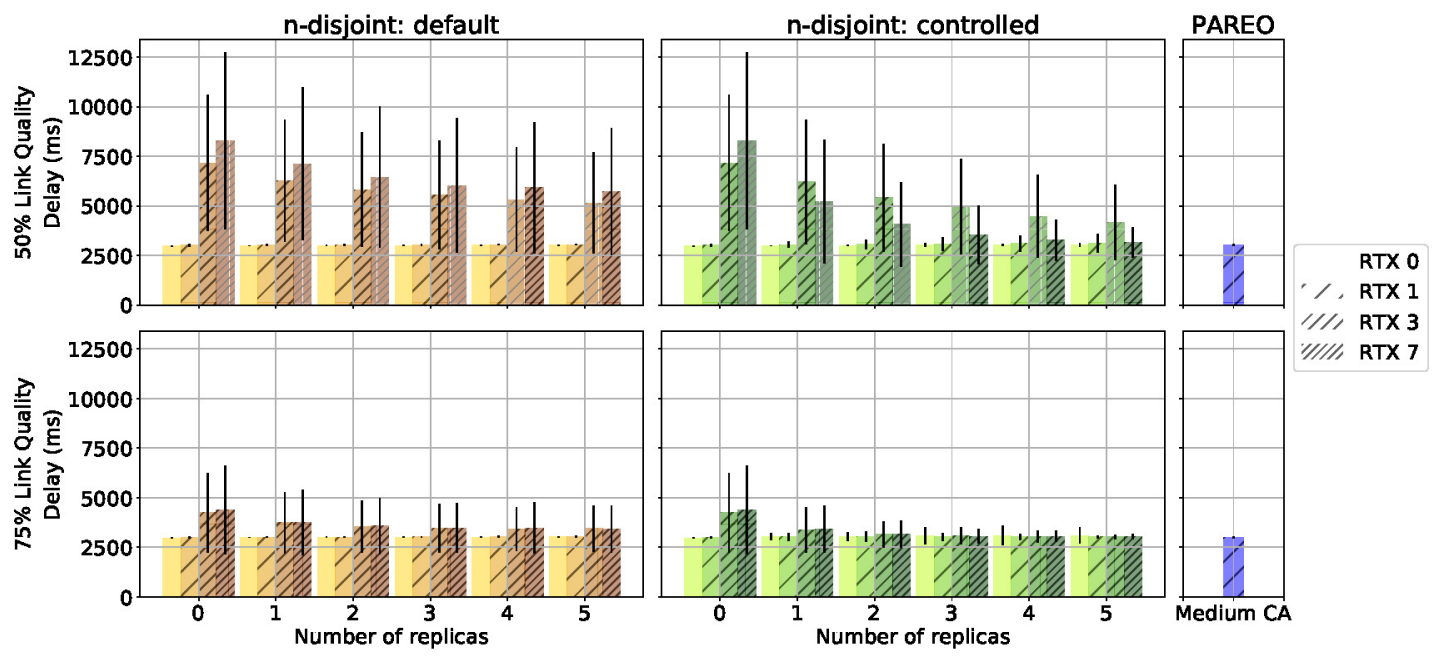

Fig. 5: Delay and jitter performances.

the jitter are significantly lower when using replications instead of retransmissions. Notice that all the replicas are sent at the same time, instead of waiting a possible acknowledgement, as it happens in the retransmission case. Furthermore, these values also get a lower variation (i.e., jitter performance) when we use both replications and retransmissions since there are higher probabilities that the packets are received. Finally, the values obtained for PAREO are equivalent to all the other schemes that have multiple replicas and single retransmission. Note that PAREO comes with the minimum jitter performance.

3) Energy Consumption: The power consumption was calculated as follows,

$$
E(P)=P_{T X} C_{T X}+P_{R X} C_{R X}+P_{\text {Idle }} C_{\text {Idle }}
$$

This case, where $C_{T X}, C_{R X}, C_{I d l e}$ are the times spent in Transmission mode (TX), Reception Mode (RX) and Idle (Idle) reported by COOJA, and $P_{T X}=52.2 \mathrm{~mW} @ 3 \mathrm{~V}$, $P_{R X}=56.4 \mathrm{~mW} @ 3 \mathrm{~V}, P_{\text {Idle }}=1.28 \mathrm{~mW} @ 3 \mathrm{~V}$ are the values for radio power consumption for the Zolertia $\mathrm{Z} 1$ mote $^{3}$ that uses the $\mathrm{CC} 2420$ radio transceiver module.

The energy consumption slightly increases with the number of replicas. As can be observed, this value gets linearly higher when retransmissions are introduced in conjunction with data packet replication. In the case of PAREO, energy consumption is higher than in the other scenarios with one retransmission; because each node that forwards a data packet has to replicate to its AP, instead of only replicating at the source, having thus in total more copies as shown in Fig. 7. Furthermore, the $\mathrm{OH}$ function has a significant impact on these values, since it requires that the nodes stay awake longer.

4) Number of Copies per Single Packet: The number of copies per packet refers to the total number of copies of one data packet that traverse the network.

The values obtained in Fig. 7 are consistent with the analytical values that we would obtain. For example, if we have seven re-

\footnotetext{
${ }^{3}$ Zolertia Z1 datasheet, Rev. C. March 2010.
}

transmissions and five replicas, we would have a total of 48 copies per level (i.e., 6 parents at the source node and 8 transmissions per parent) at most. If we need to perform the eight transmissions for all replicas, we would have a total of 288 copies. The results on n-Disjoint algorithms present in overall lower numbers because to achieve $100 \%$ PDR performance, lower retransmissions, and replicas are required. As can be observed, the number of copies increases linearly with the number of retransmissions and replicas. Note that the "n-Disjoint: default scenario" presents fewer copies because there are multiple merges of the paths in the network.

Regarding PAREO, since it comes only with one retransmission and one replication at each level, it has around 50 copies per packet in both studied cases, i.e., $50 \%$ and $75 \%$ link qualities. Furthermore, it is essential to mention that while the n-Disjoint schemes present a significantly higher number of copies when the link quality in the network drops, the PAREO solution achieves similar values.

\section{B. Discussion}

In overall, it is possible to achieve results similar to PAREO with the n-Disjoint algorithms. These results depend on the number of retransmissions and the number of replicas per packet.

Furthermore, the energy consumption of PAREO is higher, because, as stated earlier, $\mathrm{OH}$ requires that the nodes remain awake for a more extended time. However, this function also ensures a higher PDR, especially in worse link quality scenarios. Finally, we can observe that in all cases, PAREO presents better performance, especially in the $50 \%$ link quality scenario.

Finally, since there are multiple trade-offs in this study, we propose an industrial use-case with low delay and high PDR performance as requirements. As shown in Fig. 8, we compared all three algorithms with all potential configurations for the n-Disjoint schemes, i.e., 24 configurations per n-Disjoint algorithm, where each configuration is depicted as a point. The objective is to represent their PER (Packet Error Ratio) against their delay performance. The line within this figure represents the Pareto frontier, which shows the trade-off between these two 

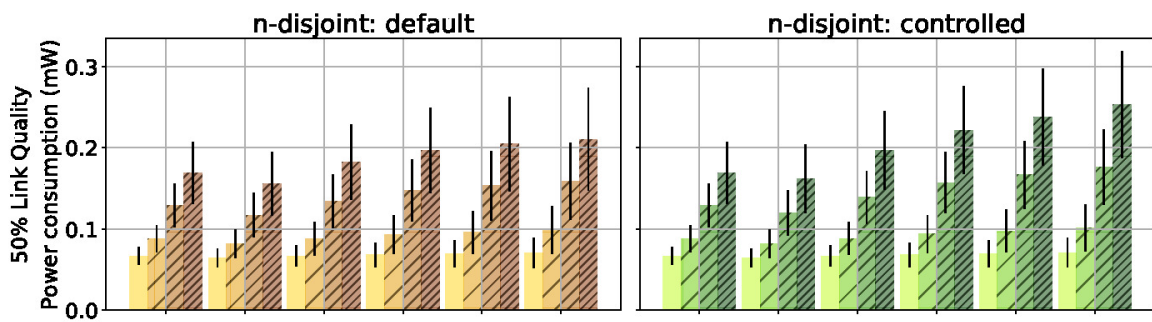

PAREO
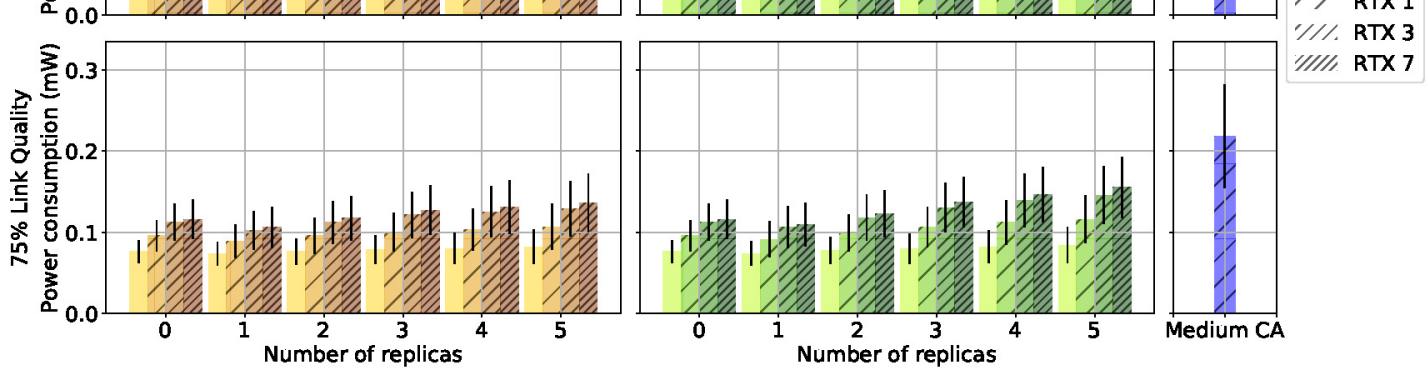

Fig. 6: Power consumption per node.
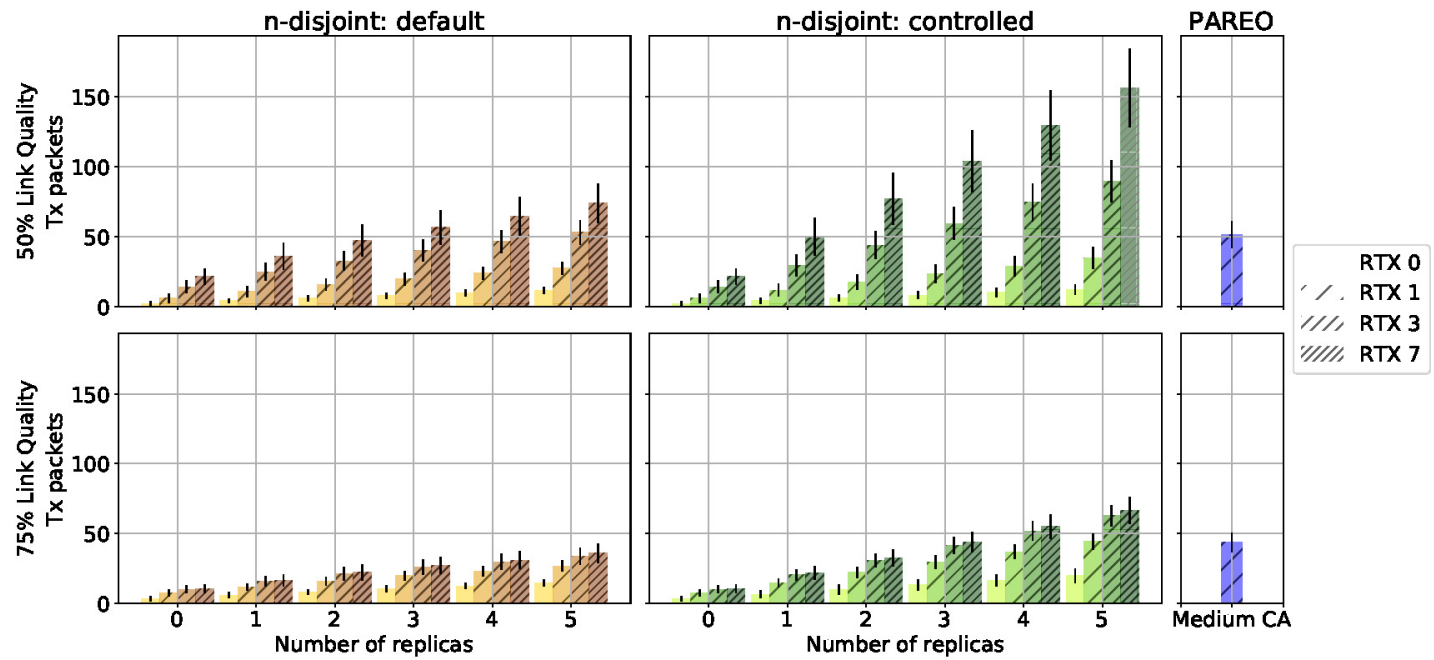

$1 / 1 /, \operatorname{RTX} 3$

Fig. 7: Total number of copies per data packet.

metrics. The compensation of a lower PER results in a higher delay and vice-versa, where the best scenario tends to get closer to the origin. We can observe that in both cases, PAREO presents the best performance, especially in the $50 \%$ link quality scenario.

\section{RELATED WORK}

Papadopoulos et al. [5] propose LeapFrog Collaboration (LFC) as an alternative for achieving predictable networking. In this paper, the Medium CA method was employed, as well as the $\mathrm{OH}$ and Packet Replication and Elimination (PRE) methods. Following this work, Koutsiamanis et al. [7] enhance the LFC analysis adding a specific TSCH schedule. This schedule is adapted to the topology used and adds a second timeslot per data transmission to ensure a lower delay. Furthermore, in [7], Koutsiamanis et al. thoroughly examine the contributions of each of the PAREO Functions, as presented in section III-C.

In [8], Jenschke et al. investigate different strategies for selecting an AP. Three algorithms are proposed in this work, the Strict CA method, the Medium CA method and the Soft CA method. There are substantial trade-offs between these strategies. Indeed, with Soft CA, the network reliability is close to $100 \%$ with the cost of energy consumption and network overhead; on the other hand, Strict CA reduces the network overhead; however, it achieves lower PDR performance.

Minet et al. [10] compare three different multipath patterns: disjoint, triangular, and braided, and analyze the trade-offs between them. The n-Disjoint algorithms proposed in this paper would be similar to the disjoint one described, while PAREO would fall under the braided category.

Ahrar et al. [11] propose a schedule aware multipath protocols that ensure low energy consumption and low delay. This schedule is optimized for braided paths, similar to the one that PAREO uses.

Lohith et al. [12] introduce LinkPeek as a separated, non-intrusive and RPL compliant mechanism that works on top of RPL or in conjunction with it instead of modifying its default behavior. When LinkPeek detects a transmission failure, it does 


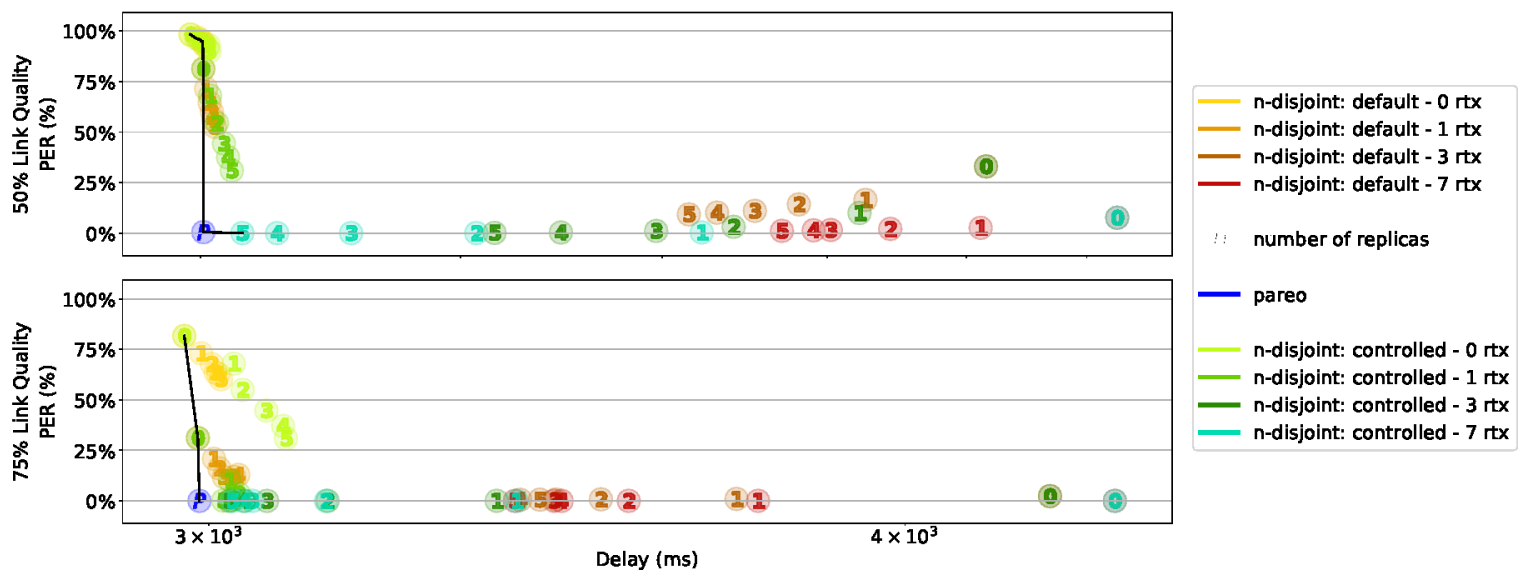

Fig. 8: Delay and PER for all studied algorithms.

a retry to the second-best parent selected by RPL, allowing for retransmission in a multipath way but lacks the delay and jitter reductions achieved by sending the copies in a parallel manner.

Finally, Armas et al. [13] investigate disjoint paths to improve network reliability. Their proposal is a subset of our n-Disjoint algorithms. However, this work comes with limited performance evaluation in terms of link quality values and network setup. Moreover, their routing configuration values set were not as complete as ours. Contrary to [13], in this work, we studied 48 different configurations of the n-Disjoint schemes, and we evaluated with different link qualities (i.e., $50 \%$ and $75 \%$ ), as well as we compared our algorithms against PAREO Functions.

\section{CONCLUSION}

In this paper, we proposed two different algorithms based on disjoint paths: "n-Disjoint paths: default scenario" and " $n$ Disjoint paths: controlled scenario," delivering quality-of-service through multipath routing. Both algorithms employ the concept of packet replication that improves the reliability of the network without compromising the delivery times, and retransmissions that provide more opportunities for the packet transmissions.

Furthermore, we compared our proposed mechanism with the PAREO solution that offers a state-of-the-art method for doing multipath in LLNs. Our thorough performance evaluation campaign demonstrates that PAREO Functions present a better performance in terms of reliability and jitter. This performance improvement can be seen especially in lower link qualities scenarios since it concentrates its opportunities as it tends to select its PP and its AP in a specific direction. n-Disjoint schemes, on the other hand, require less energy to achieve a similar PDR performance at the cost of a higher number of copies per packet.

\section{ACKNOWLEDGMENTS}

Experiments presented in this paper were carried out using the Grid' 5000 testbed, supported by a scientific interest group hosted by INRIA and including CNRS, RENATER and several Universities as well as other organizations (see https://www.grid5000.fr). Additionally, this work was partially performed and supported under the TPI ANR17-CE10-0007-01 project of the French National Research Agency and by the UBACyT 20020170100421BA project of the Universidad de Buenos Aires.

\section{REFERENCES}

[1] K. Kritsis, G. Z. Papadopoulos, A. Gallais, P. Chatzimisios, and F. Theoleyre, "A Tutorial on Performance Evaluation and Validation Methodology for Low-Power and Lossy Networks," IEEE Communications Surveys and Tutorials, vol. 20, pp. 1799-1825, 2018.

[2] T. Winter, P. Thubert, A. Brandt, J. Hui, R. Kelsey, P. Levis, K. Pister, R. Struik, J. Vasseur, and A. R., "RPL: IPv6 Routing Protocol for Low-Power and Lossy Networks," IETF RFC 6550, 2012.

[3] P. Thubert, "Objective Function Zero for the Routing Protocol for Low-Power and Lossy Networks (RPL)," RFC 6552, Mar. 2012.

[4] "IEEE Standard for Low-Rate Wireless Personal Area Networks (LR-WPANs)," IEEE Std 802.15.4-2015 (Revision of IEEE Std 802.15.4-2011), April 2016.

[5] G. Z. Papadopoulos, T. Matsui, P. Thubert, G. Texier, T. Watteyne, and N. Montavont, "Leapfrog Collaboration: Toward Determinism and Predictability in Industrial-IoT applications," in Proceedings of the IEEE International Conference on Communications (ICC), 2017.

[6] R. A. Koutsiamanis, G. Z. Papadopoulos, X. Fafoutis, J. M. Del Fiore, P. Thubert, and N. Montavont, "From Best-Effort to Deterministic Packet Delivery for Wireless Industrial IoT Networks," IEEE Transactions on Industrial Informatics, vol. 14, pp. 4468 - 4480, 2018.

[7] R.-A. Koutsiamanis, G. Papadopoulos, T. Lagos Jenschke, P. Thubert, and N. Montavont, "Meet the PAREO Functions: Towards Reliable and Available Wireless Networks," in IEEE International Conference on Communications (ICC), Dublin, Ireland, Jun. 2020.

[8] T. L. Jenschke, G. Z. Papadopoulos, R. Koutsiamanis, and N. Montavont, "Alternative Parent Selection for Multi-Path RPL Networks," in Proceedings of the IEEE 5th World Forum on Internet of Things (WF-IoT), Limerick, Ireland, April 2019.

[9] D. Barthel, J. Vasseur, K. Pister, M. Kim, and N. Dejean, "Routing Metrics Used for Path Calculation in Low-Power and Lossy Networks," RFC 6551, Mar. 2012.

[10] P. Minet, I. Khoufi, and A. Laouiti, "Increasing Reliability of a TSCH Network for the Industry 4.0," In Proceedings of the I6th IEEE International Symposium on Network Computing and Applications (NCA), 2017.

[11] E. M. Ahrar, M. Nassiri, and F. Theoleyre, "Multipath aware scheduling for high reliability and fault tolerance in low power industrial networks," in Journal of Network and Computer Applications, September 2019, pp. 25-36.

[12] Y. S. Lohith, T. S. Narasimman, S. V. R. Anand, and M. Hedge, "Link peek: A link outage resilient ip packet forwarding mechanism for 6lowpan/rpl based low-power and lossy networks (llns)," in 2015 IEEE International Conference on Mobile Services, 2015, pp. 65-72.

[13] J. d. Armas, P. Tuset, T. Chang, F. Adelantado, T. Watteyne, and $\mathrm{X}$. Vilajosana, "Determinism through path diversity: Why packet replication makes sense," in 2016 International Conference on Intelligent Networking and Collaborative Systems (INCoS), 2016, pp. 150-154. 